\title{
Expressing Numbers in terms of Golden, Silver and Bronze Ratios
}

\section{Dr. R. Sivaraman ${ }^{1}$}

${ }^{1}$ Associate Professor, Department of Mathematics, D. G. Vaishnav College, Chennai, India,National Awardee for Popularizing Mathematics among masses rsivaraman1729@yahoo.co.in Article History: Received: 11 January 2021; Accepted: 27 February 2021; Published online: 5 April 2021

Abstract: The idea of expressing certain kind of numbers as linear combination of special class of numbers has always been an interesting exercise in mathematics. In this paper, I present an interesting way to write a given natural number as sum or difference of integral powers of golden ratio, silver ratio and bronze ratio. Suitable illustrations enabling the process are briefed in the paper. Keywords: Recurrence Relation, Golden Ratio, Silver Ratio, Bronze Ratio, Mathematical Induction.

\section{Introduction}

Leonardo Fibonacci of Italy published his famous book Liber Abaci in 1202 CE. This book was instrumental in introducing Indian numerals to entire world. Apart from various interesting problems it possessed, the problem on growth of immortal rabbits paved way for describing the most famous Fibonacci numbers, the sequence of numbers named after him. A slight modification of Fibonacci sequence leads to a sequence called Lucas Sequence. It is well known that the ratio of successive Lucas numbers approaches to Golden Ratio, which has so much of significance not only in mathematics but also in Architecture and Engineering. In this paper, I will prove some important results for expressing any natural number as sum or difference of integral powers of gold, silver and bronze ratios.

\section{Definitions}

2.1 The Lucas Sequence is defined recursively by the sequence $L_{n+2}=L_{n+1}+L_{n}, n \geq 0$ (2.1) where $L_{0}=2, L_{1}=1$. Using (2.1), the terms of the Lucas sequence are given by $2,1,3,4,7,11,18,29,47,76,123,199, \ldots$ We observe that except the first two terms, each term is sum of two preceding terms.

If $L_{n}$ is the $n$th Lucas number then we know that (see [1]) $\frac{L_{n+1}}{L_{n}} \rightarrow \varphi$ as $n \rightarrow \infty$. That is, the ratio of successive terms of Lucas sequence approaches a number $\varphi=\frac{1+\sqrt{5}}{2}$ called Golden Ratio.

2.2 We define sequence recursively by $P_{n+2}=2 P_{n+1}+P_{n}, n \geq 1$ (2.2) where $P_{0}=1, P_{1}=2, P_{2}=6$. Using (2.2), the terms of the sequence are given by $1,2,6,14,34,82,198,478,1154, \ldots$

We observe that except for the first three terms, each term is obtained by adding twice the previous term and last but one term.

If $P_{n}$ is the $n$th term of the sequence described then we know that (see [1]) $\frac{P_{n+1}}{P_{n}} \rightarrow \lambda$ as $n \rightarrow \infty$ where $\lambda=1+\sqrt{2}$ is called Silver Ratio.

2.3 We define a sequence recursively by $B_{n+2}=3 B_{n+1}+B_{n}, n \geq 1$ (2.3) where $B_{0}=1, B_{1}=3, B_{2}=11$. Using (2.3), the terms of the sequence are given by $1,3,11,36,119,393,1298, \ldots$ We observe that except for the first three terms, each term is obtained by adding thrice the previous term and last but one term.

If $B_{n}$ is the $n$th term of the sequence described then we know that (see [1]) $\frac{B_{n+1}}{B_{n}} \rightarrow \mu$ as $n \rightarrow \infty$ where $\mu=\frac{3+\sqrt{13}}{2}$ is called Bronze Ratio.

\section{Integral Powers of Golden Ratio}

In the following theorem, I will relate sum of integral powers of golden ratios to Lucas numbers. 


\subsection{Theorem 1}

If $\varphi$ is the golden ratio and $L_{n}$ is the $n$th Lucas number then $\varphi^{n}+\left(\frac{-1}{\varphi}\right)^{n}=L_{n}, n \geq 0$ (3.1)

Proof: In view of (2.1), we obtain the quadratic equation $x^{2}-x-1=0(3.2)$. We notice that the two real roots of (3.2) are given by $\varphi=\frac{1+\sqrt{5}}{2}, \frac{-1}{\varphi}=\frac{1-\sqrt{5}}{2}$. The sum of roots is $\varphi-\frac{1}{\varphi}=1(3.3)$

We shall prove the theorem by induction on $n$. For $n=0, \varphi^{0}+\left(-\frac{1}{\varphi}\right)^{0}=2=L_{0}$.

If $n=1$, then equation (3.1) can be expressed in the form $\varphi+\left(-\frac{1}{\varphi}\right)=\left(\frac{1+\sqrt{5}}{2}\right)+\left(\frac{1-\sqrt{5}}{2}\right)=1=L_{1}$

Since $\varphi-\frac{1}{\varphi}=1$, we have $\varphi^{2}+\frac{1}{\varphi^{2}}=3=L_{2}$. Thus equation (3.1) is true for the values $n=0,1,2$.

Now by Induction Hypothesis, we will assume that (3.1) holds true for all values of $n$ up to some natural number $k$ and we will prove the result for $n=k+1$.

Thus, we assume that $\varphi^{n}+\left(-\frac{1}{\varphi}\right)^{n}=L_{n}, n=1,2,3, \ldots, k(3.4)$.

$\left[\varphi^{k}+\left(-\frac{1}{\varphi}\right)^{k}\right] \times\left[\varphi-\frac{1}{\varphi}\right]=\left[\varphi^{k+1}+\left(-\frac{1}{\varphi}\right)^{k+1}\right]-\left[\varphi^{k-1}+\left(-\frac{1}{\varphi}\right)^{k-1}\right]$

Using (2.1), (3.3) and (3.4), we have

$\varphi^{k+1}+\left(-\frac{1}{\varphi}\right)^{k+1}=L_{k}+L_{k-1}=L_{k+1}$

Thus the result is true for $n=k+1$ whenever it is true up to $n=k$. Hence, by Induction Principle, equation (3.1) is true for all natural numbers $n$. This completes the proof.

\subsection{Theorem 2}

Any natural number can be expressible as sum or difference of integral powers of golden ratio.

Proof: By Zeckendorf's theorem we know that any natural number can be expressed uniquely as a sum of non-consecutive Lucas numbers. By (3.1), we know that for any natural number $n$, the $n$th Lucas number is expressible as $\varphi^{n}-\varphi^{-n}$ if $n$ is odd and $\varphi^{n}+\varphi^{-n}$ if $n$ is even. Hence any natural number can be expressible as sum or difference of integral powers of golden ratio. This completes the proof.

\subsection{Illustrations}

In this section, I will demonstrate the technique of actually expressing any natural number as sum or difference of integral powers of golden ratio (integer powers of golden ratio).

If we consider the number 12, the number of months in any year, then first we will try to express 12 as sum of terms of Lucas sequence. Doing so, we get $12=1+11$. Now using (3.1), we have $12=1+11=L_{1}+L_{5}=\left(\varphi-\frac{1}{\varphi}\right)+\left(\varphi^{5}-\frac{1}{\varphi^{5}}\right)=\varphi-\varphi^{-1}+\varphi^{5}-\varphi^{-5}$.

Similarly, for 1729 we have, $1729=3+11+29+322+1364=L_{2}+L_{5}+L_{7}+L_{12}+L_{15}$. Thus using (3.1), we have $1729=\varphi^{2}+\varphi^{-2}+\varphi^{5}-\varphi^{-5}+\varphi^{7}-\varphi^{-7}+\varphi^{12}+\varphi^{-12}+\varphi^{15}-\varphi^{-15}$.

Similar to these illustrations, given any natural number, first we try to express that sum of Lucas numbers and using (3.1), we can express as sum or difference of integral powers of golden ratio.

\section{Integral Powers of Silver Ratio}


In the following theorem, I will relate sum of integral powers of silver ratios to the terms of the sequence defined in 2.2

\subsection{Theorem 3}

If $\lambda=1+\sqrt{2}$ is the silver ratio and if $P_{n}$ is the $n$th term of the sequence defined through recurrence relation (2.2), then $\lambda^{n}+\left(-\frac{1}{\lambda}\right)^{n}=P_{n}, n \geq 1$

Proof: Through the recurrence relation (2.2) we obtain the quadratic equation $x^{2}-2 x-1=0$ (4.2). The two real roots of this quadratic equation are given by $\lambda=1+\sqrt{2},-\frac{1}{\lambda}=1-\sqrt{2}$. The sum of the roots is $\lambda-\frac{1}{\lambda}=2$ (4.3). We shall prove the theorem by induction on $n$. If $n=1$, then equation (4.1) can be expressed in the form

$\lambda+\left(-\frac{1}{\lambda}\right)=\lambda-\frac{1}{\lambda}=2=P_{1}$. Similarly, since $\lambda-\frac{1}{\lambda}=2$ we have $\lambda^{2}+\frac{1}{\lambda^{2}}=6=P_{2}$. Thus the result is true for $n=1,2$.

By Induction Hypothesis, we will assume that (4.1) holds true for all values of $n$ up to some natural number $k$ and we will prove the result for $n=k+1$.

Thus, we assume that $\lambda^{n}+\left(-\frac{1}{\lambda}\right)^{n}=P_{n}$ for $n=1,2,3, \ldots, k$

$\left[\lambda^{k}+\left(-\frac{1}{\lambda}\right)^{k}\right] \times\left(\lambda-\frac{1}{\lambda}\right)=\left[\lambda^{k+1}+\left(-\frac{1}{\lambda}\right)^{k+1}\right]-\left[\lambda^{k-1}+\left(-\frac{1}{\lambda}\right)^{k-1}\right]$

Using (2.2), (4.3) and (4.4), we have $\left[\lambda^{k+1}+\left(-\frac{1}{\lambda}\right)^{k+1}\right]=2 P_{k}+P_{k-1}=P_{k+1}$.

Thus if the result is true for all values up to $k$, then it is also true for $k+1$. Hence by Induction Principle, (4.1) is true for all natural numbers $n$. This completes the proof.

\subsection{Theorem 4}

Any natural number can be expressible as sum or difference of integral powers of silver ratio.

Proof: First, we note that 1 can be written as integral power of silver ratio in the form $\lambda^{0}=1=P_{0}$. Now considering any natural number, we can express it as sum or difference of terms of the sequence defined in (2.2). Now, by theorem 3 , we know that the $n$th term $(n \geq 1)$ of this sequence namely $P_{n}$ can be expressed as $\lambda^{n}-\lambda^{-n}$ if $n$ is odd and $\lambda^{n}+\lambda^{-n}$ if $n$ is even. Hence any natural number except 1 can be expressible as sum or difference of integral powers of silver ratio. This completes the proof.

\subsection{Illustrations}

In this section, I will consider four natural numbers greater than 1 and try to express them as sum or difference of integral powers of silver ratio. The terms of the sequence corresponding to (2.3) are given by $1,2,6,14,34,82,198,478,1154,2786,6726,16238,39202,94642,228486, \ldots$

If we consider 12, then using the terms of the sequence defined in (2.2) and (4.1), we have

$12=14-2=P_{3}-P_{1}=\lambda^{3}-\lambda^{-3}-\lambda+\lambda^{-1}$

If we consider the numbers 1729,3435 and 142857 then using same principle we have

$$
\begin{aligned}
1729 & =1+14+82+478+1154=P_{0}+P_{3}+P_{5}+P_{7}+P_{8} \\
& =\lambda^{0}+\lambda^{3}-\lambda^{-3}+\lambda^{5}-\lambda^{-5}+\lambda^{7}-\lambda^{-7}+\lambda^{8}+\lambda^{-8}
\end{aligned}
$$




$$
\begin{aligned}
& 3435=1+6-34+198+478+2786=P_{0}+P_{2}-P_{4}+P_{6}+P_{7}+P_{9} \\
& =\lambda^{0}+\lambda^{2}+\lambda^{-2}-\lambda^{4}-\lambda^{-4}+\lambda^{6}+\lambda^{-6}+\lambda^{7}-\lambda^{-7}+\lambda^{9}-\lambda^{-9} \\
& 142857=94642+39202+6726+2786-478-82-34-6+1 \\
& =\lambda^{13}-\lambda^{-13}+\lambda^{12}+\lambda^{-12}+\lambda^{10}+\lambda^{-10}+\lambda^{9}-\lambda^{-9}-\lambda^{7}+\lambda^{-7}-\lambda^{5}+\lambda^{-5}-\lambda^{4}-\lambda^{-4}-\lambda^{2}-\lambda^{-2}+\lambda^{0}
\end{aligned}
$$

These illustrations verify theorem 4 established above.

\section{Integral Powers of Bronze Ratio}

In the following theorem, I will relate sum of integral powers of bronze ratios to the terms of the sequence defined in 2.3

\subsection{Theorem 5}

If $\mu=\frac{3+\sqrt{13}}{2}$ is the bronze ratio and if $B_{n}$ is the $n$th term of the sequence defined through recurrence relation (2.3), then $\mu^{n}+\left(-\frac{1}{\mu}\right)^{n}=B_{n}, n \geq 1$

Proof: Using the recurrence relation (2.3), we obtain the quadratic equation $x^{2}-3 x-1=0$

whose roots are $\mu=\frac{3+\sqrt{13}}{2},-\frac{1}{\mu}=\frac{3-\sqrt{13}}{2}$. The sum of roots is $\mu-\frac{1}{\mu}=3$ (5.3). We shall prove the theorem by induction on $n$. If $n=1$, then equation (5.1) can be expressed in the form

$\mu+\left(-\frac{1}{\mu}\right)=\mu-\frac{1}{\mu}=3=B_{1}$. Similarly, since $\mu-\frac{1}{\mu}=3$ we get $\mu^{2}+\frac{1}{\mu^{2}}=11=B_{2}$. Thus the result is true for $n=1,2$. We will assume the result to be true up to $n=1,2,3, \ldots, k$ and prove for $\quad n=k+1$.

Thus, we assume that $\mu^{n}+\left(-\frac{1}{\mu}\right)^{n}=B_{n}$ for $n=1,2,3, \ldots, k$ (5.4)

$$
\left[\mu^{k}+\left(-\frac{1}{\mu}\right)^{k}\right] \times\left(\mu-\frac{1}{\mu}\right)=\left[\mu^{k+1}+\left(-\frac{1}{\mu}\right)^{k+1}\right]-\left[\mu^{k-1}+\left(-\frac{1}{\mu}\right)^{k-1}\right]
$$

Using (2.3), (5.3) and (5.4), we have $\left[\mu^{k+1}+\left(-\frac{1}{\mu}\right)^{k+1}\right]=3 B_{k}+B_{k-1}=B_{k+1}$.

Thus if the result is true for all values up to $k$, then it is also true for $k+1$. Hence by Induction Principle, (5.1) is true for all natural numbers $n$. This completes the proof.

\subsection{Theorem 6}

Any natural number can be expressible as sum or difference of integral powers of bronze ratio with two repetitions of first term 1 of the sequence defined in (2.3)

Proof: First, we note that 1 can be written as integral power of bronze ratio in the form $\mu^{0}=1=B_{0}$. Now considering any natural number, we can express it as sum or difference of terms of the sequence defined in (2.3). with possibly two repetitions of first term namely 1 , if required. For example, $5=3+1+1=B_{1}+B_{0}+B_{0}$. Now, by theorem 5 , we know that $n$th term $\quad(n \geq 1)$ of the sequence $B_{n}$ for can be expressed as $\mu^{n}-\mu^{-n}$ if $n$ is odd and $\mu^{n}+\mu^{-n}$ if $n$ is even. Hence any natural number can be expressible as sum or difference of integral powers of bronze ratio with two repetitions (if required) of first term 1 . This completes the proof. 


\subsection{Illustrations}

In this section, I will consider two natural numbers and try to express them as sum or difference of integral powers of bronze ratio. The terms of the sequence corresponding to (2.3) are given by $1,3,11,36,119,393,1298,4287,17148,68592,222924, \ldots$

$1729=1298+393+36+3-1=B_{6}+B_{5}+B_{3}+B_{1}-B_{0}$

$$
=\mu^{6}+\mu^{-6}+\mu^{5}-\mu^{-5}+\mu^{3}-\mu^{-3}+\mu-\mu^{-1}-\mu^{0}
$$

We notice for expressing 1729 there is no need for the repetition of the first term 1. Now I consider the number 142857 and try to obtain the required form.

$$
142857=222924-68592-17148+4287+1298+119-36+3+1+1
$$$$
=\mu^{10}+\mu^{-10}-\mu^{9}+\mu^{-9}-\mu^{8}-\mu^{-8}+\mu^{7}-\mu^{-7}+\mu^{6}+\mu^{-6}+\mu^{4}+\mu^{-4}-\mu^{3}+\mu^{-3}+\mu-\mu^{-1}+\mu^{0}+\mu^{0}
$$

In this case, we notice that the constant term $1=\mu^{0}$ gets repeated twice.

These illustrations verify theorem 6 established above.

\section{Conclusion}

The concept of expressing any natural number as sum or difference of integer powers of golden, silver and bronze ratios has been proved in this paper. This has been done using theorems 2,4 and 6 respectively. While for golden and silver ratios, there is no need for repetition of any terms of the corresponding sequences considered, for bronze ratio, in some cases we require the repletion of the first term namely 1 twice to achieve the decomposition of the given natural number as sum or difference of integer powers of bronze ratio.

We can extend this process for other metallic ratios also but in that case we need more repetitions of the terms of the sequence describing them, since the terms of general metallic ratio sequence gets widely distributed. Hence, I just considered the first three metallic ratios in this paper.

\section{REFERENCES}

1. R. Sivaraman, Exploring Metallic Ratios, Mathematics and Statistics, Horizon Research Publications, (Scopus Indexed Journal), Volume 8, Issue 4, (2020), 388 - 391.

2. Juan B. Gil and Aaron Worley, Generalized Metallic Means, Fibonacci Quarterly, Volume 57 (2019), Issue. 1, 45-50.

3. Dann Passoja, Reflections on the Gold, Silver and Metallic Ratios, 2015.

4. Krcadinac V., A new generalization of the golden ratio. Fibonacci Quarterly, 2006;44(4):335-340.

5. P. J. Cameron, Some sequences of integers, Discrete Math.75 (1989), 89-102.

6. K. Hare, H. Prodinger, and J. Shallit, Three series for the generalized golden mean, Fibonacci Quart. 52(2014), no. 4, 307-313. 